\title{
Parameters and models of the jets from Sanduleak's star in the LMC
}

\author{
A. Camps-Fariña ${ }^{1}$, A. C. Raga ${ }^{1}$, and A. Noriega-Crespo ${ }^{2}$ \\ ${ }^{1}$ Instituto de Ciencias Nucleares, Universidad Nacional Autónoma de México, Ap. 70-543, 04510 Cd. Mx., Mexico \\ e-mail: artemi.camps@correo.nucleares.unam.mx; raga@nucleares.unam.mx \\ ${ }^{2}$ Space Telescope Science Institute, 3700 San Martin Drive, Baltimore, MD 21218, USA
}

Received 2 March 2018 / Accepted 7 August 2018

\begin{abstract}
Context. Angeloni and collaborators have discovered a bipolar jet extending out to $\sim 6 \mathrm{pc}$ from "Sanduleak's star" in the Large Magellanic Cloud (LMC). This is the first angularly resolved stellar jet system that has been observed outside our Galaxy.

Aims. In this paper we use archival Hubble Space Telescope (HST) images of this jet in order to estimate the flow parameters, and use them to explore two possible theoretical scenarios for modelling this bipolar outflow.

Methods. We have computed axisymmetric gasdynamic simulations of a conical outflow with an opening angle that increases with time and of a cylindrical jet interacting with a stratified circumstellar structure. The results of these models are then compared with the observed morphology of the jets from Sanduleak's star.

Results. From the observations, we obtain $\dot{M}_{j} \approx 1.2 \times 10^{-5} M_{\odot} \mathrm{yr}^{-1}$ and $L_{\mathrm{m}} \approx 1000 L_{\odot}$ for the mass loss rate and the mechanical luminosity (respectively) of each of the outflow lobes. We also obtain a (deprojected) flow velocity $v_{j} \approx 1000 \mathrm{~km} \mathrm{~s}^{-1}$ and a dynamical time $t_{\mathrm{dyn}} \approx 7300 \mathrm{yr}$. From the simulations that we have computed (with these flow parameters), we find that both the "opening out conical wind" and the "jet+stratified environment" scenarios have characteristics that resemble the observed morphologies. A more complete model for the jets from Sanduleak's star might incorporate some of the features of these two scenarios.
\end{abstract}

Key words. stars: winds, outflows - ISM: jets and outflows - stars: individual: Sanduleak's star

\section{Introduction}

Sanduleak's star is a remarkable compact emission-line object in the Large Magellanic Cloud (LMC). Sanduleak (1977) found a variability in the $\mathrm{H} \alpha$ emission of this object, and suggested that it might be a symbiotic star. Allen (1980) obtained optical/IR spectra and photometry of this object, detecting a number of emission lines and a flat continuum, which they suggest might be of nebular origin. The steep Balmer line progression implies $A_{V} \sim 2-3 \mathrm{mag}$ extinction. Kafatos et al. (1983) present optical and ultraviolet (IUE) spectra, showing lines from ionization states ranging from the [O I] 6300 line to the N V 1238, 1242 lines. These authors note that the strong [N II] lines observed in this object are reminiscent of some regions of the $\eta$ Carina nebula (e.g., the "jet region" observed by Meaburn et al. 1993), which show evidence for strong $\mathrm{N}$ enrichment, and conclude that it is not clear whether Sanduleak's star is a symbiotic star or an object resembling $\eta$ Carina.

Angeloni et al. (2011) present the discovery of a remarkable bipolar jet system ejected from Sanduleak's star. The two outflow lobes have an angular extent of $\approx 1^{\prime}$ (corresponding to $\sim 15 \mathrm{pc}$ at a $50 \mathrm{kpc}$ distance to the LMC; see Schaefer 2008). These authors present a long-slit spectrum which shows an outward "acceleration" of the radial velocities, reaching values of approximately $\pm 400 \mathrm{~km} \mathrm{~s}^{-1}$ (with respect to the systemic velocity, with blue-shifted velocities to the W) at the heads of the outflow lobes.

They argue that this jet system is likely oriented close to the plane of the sky, such that the outflow velocities are substantially higher. Angeloni et al. (2011) note that this object is the first angularly resolved, extragalactic stellar jet that has been observed, and that it should be modelled theoretically in order to understand its similarities and differences with respect to jets associated with (Galactic) young stars and/or planetary nebulae.

We should mention that a second bipolar stellar jet in the LMC (HH 1177) has recently been reported by Macleod et al. (2018). This jet system appears to be ejected from a young star with a $\sim 10 M_{\odot}$ mass.

The following are the two objects that morphologically resemble the jets from Sanduleak's star:

- Fleming 1: a PN with a bipolar outflow with a remarkable precession signature (see, e.g., Palmer et al. 1996),

- R Aquarii: a symbiotic star with a bipolar, jet-like outflow (see, e.g., Melnikov et al. 2018).

Clearly, the theoretical ideas that have been proposed for modelling these objects are possibilities for modelling the jets from Sanduleak's star, see Palmer et al. (1996) and Bugarella et al. (1992).

In this paper, we present attempts to model the jet from Sanduleak's star with two different flow configurations:

1. a conical outflow of increasing opening angle as a function of time,

2. a cylindrical jet interacting with a radially stratified structure around the star.

The first of these flows has been studied by Cantó et al. (2008) to model the bipolar cavities around Barnard 5 IRS 1 (observed by Velusamy \& Langer 1998). This object shows lobes with a curved cavity close to the source plus an axial emission at larger distances, which is reminiscent of the outflow from 

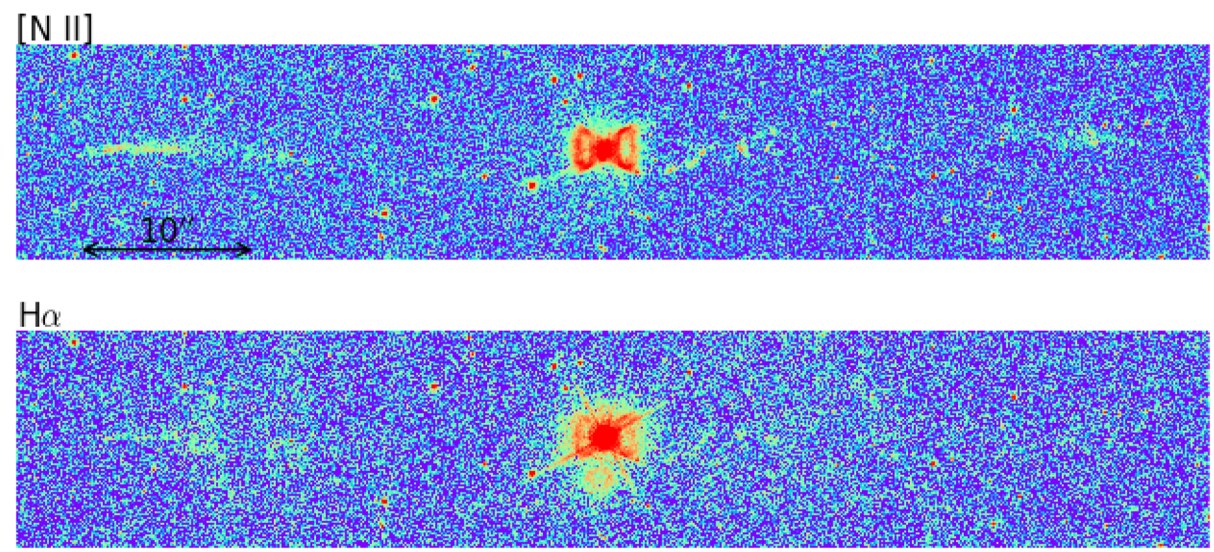

Fig. 1. [N II] (top panel) and $\mathrm{H} \alpha$ (bottom panel) HST images of the jets from Sanduleak's star. The images have been rotated to place the outflow axis (with PA = $71^{\circ}$ ) along the abscissa. The abscissa approximately points to the WSW and the ordinate to the NNW. The calibrated images are shown with the logarithmic colour scale given (in erg $\mathrm{s}^{-1} \mathrm{~cm}^{-2} \operatorname{arcesc}^{-2}$ ) by the bottom bar. A constant background of $1.5 \mathrm{erg} \mathrm{s}^{-1} \mathrm{~cm}^{-2} \operatorname{arcsec}^{-2}$ has been subtracted $10^{-16}$ $10^{-15}$ $10^{-14}$ from the $\mathrm{H} \alpha$ image.

Sanduleak's star. In the case of the outflow from Sanduleak's star, the stratified structure surrounding the outflow source could be a low-velocity wind from a binary companion of the outflow source (see Soker 2005 for a discussion of the binarity of $\eta$ Carinae). The second of these flows has been studied by Lloyd et al. (1993) to model recurrent novae with jet-like features (see, e.g., Harvey et al. 2016).

The paper is organized as follows. In Sect. 2, we use archival HST $\mathrm{H} \alpha$ and [N II] 6583 images of the region around Sanduleak's star to show the morphology of the jet system and to estimate its physical parameters (emission line luminosity, density, orientation angle, size, mass loss rate and mechanical luminosity). Section 3 describes the hydrodynamic code and the configuration used to model the conical outflows (see point 1, above) and the computed simulations. In Sect. 4, we describe the configuration used to compute jet+stratified envelope models (see point 2, above) and the resulting simulations. The results are then summarized in Sect. 5.

\section{The jet parameters}

\subsection{The HST archival images}

From the HST archive, we have downloaded three images of the region around Sanduleak's star (from proposal 12868, led by Rodolfo Angeloni) obtained with the F656N (H $\alpha$ ), F658N ([N II] 6583) and F6730 ([S II] 6716+30) narrow-band filters. The jets from Sanduleak's star are detected in the F656N (2672 s exposure) and F658N (2862 s) frames, but not in the F673N (3139 s) frame. Therefore, in the following we only use the F656N and F658N frames (to which we refer as the $\mathrm{H} \alpha$ and $[\mathrm{N}$ II] frames, respectively). The frames have been obtained with the WFC3 camera, and have a $0.0396^{\prime \prime} /$ pix sampling. We have used the information in the header to obtain a flux calibration.

Figure 1 shows the [N II] and $\mathrm{H} \alpha$ frames, rotated such that the abscissa is parallel to the outflow axis, which has a PA $=71^{\circ}$ ENE jet and an oppositely directed counterjet (see Angeloni et al. 2011). It is clear that in these frames the region within $\sim 5^{\prime \prime}$ from the source is well detected, but the emission at larger distances is barely visible above the noise.

Because of this, we have computed images with an adaptive smoothing, in which the outflow can be better seen. This is described in the following section.

\subsection{Smoothed images}

In order to preserve the structure of the high-emission regions while improving the detection for the low-emission features, we need to process the image with different degrees of smoothing depending on surface brightness.

The basis we used to determine the varying kernel sizes over the image was to first apply Voronoi binning. This procedure calculates bins of different size such that each bin has at least a target signal-to-noise ratio $(\mathrm{S} / \mathrm{N})$. In this manner, high surface brightness areas have small bins while fainter emission pixels are grouped into larger bins. We applied the method from Cappellari \& Copin (2003) which performs this calculation for 2D data, as is the case with our images.

First of all, we used SEXTRACTOR (Bertin \& Arnouts 1996) to detect and then mask the foreground stars, as they would interfere with the binning procedure, and then performed the binning to various $\mathrm{S} / \mathrm{N}$ values. The best improvement of the images was obtained with target $\mathrm{S} / \mathrm{N}$ of 10 for $[\mathrm{N} \mathrm{II}]$ and 15 for $\mathrm{H} \alpha$. This corresponds to larger bins and therefore more smoothing in $\mathrm{H} \alpha$ owing to the higher target $\mathrm{S} / \mathrm{N}$, which means that more pixels need to be aggregated to meet the target value.

We then created a map of the bin sizes for each image by first assigning the equivalent radius of each bin to its centre coordinates and then used a cubic interpolation to produce the map. We did this instead of assigning the size of each bin to all the pixels it encompasses in order to avoid edge effects between bins which would appear in the smoothed images. Finally, we produced the smoothed images by applying a Gaussian kernel to each pixel with a width obtained from the bin size map. In Fig. 2 we show the intermediate steps for obtaining the smoothed images.

The resulting smoothed [N II] and $\mathrm{H} \alpha$ maps are shown in Fig. 3. The emitting structures are more clearly seen in the [N II] (top) map:

- a bright "limb-brightened cavity" (or "hourglass") structure within $\sim 5^{\prime \prime}$ from the outflow source,

- elongated, axial, emitting regions (in the $\mathrm{E}$ and $\mathrm{W}$ lobes) extending from $\sim 15^{\prime \prime} \rightarrow 30^{\prime \prime}$ from the source,

- traces of emitting structures which seem to be joining the outer edge of the inner, hourglass nebula to the outer, axial emitting regions. This is seen particularly clearly in the sourthern region of the $\mathrm{W}$ lobe of the hourglass nebula. 


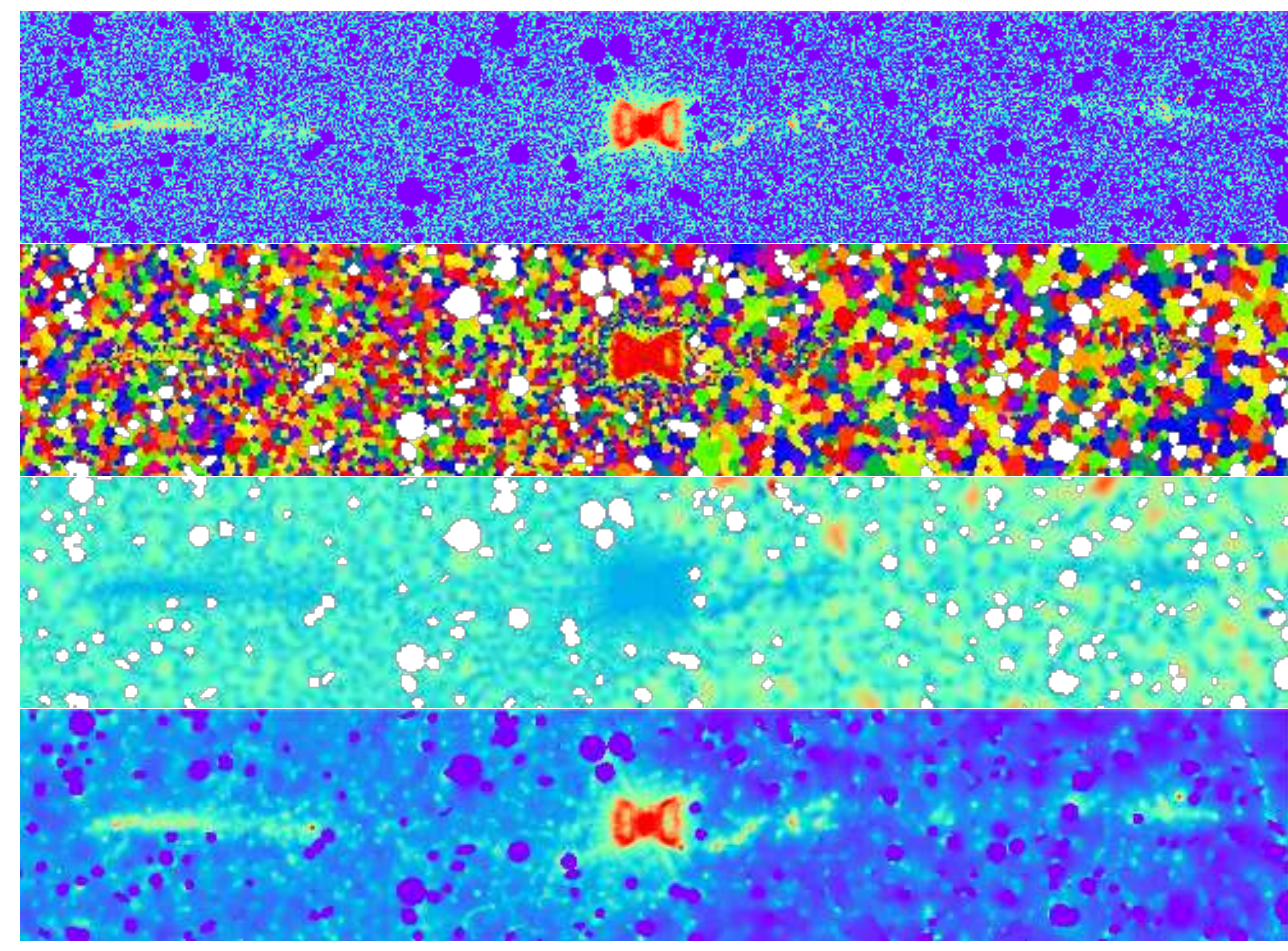

[N II]

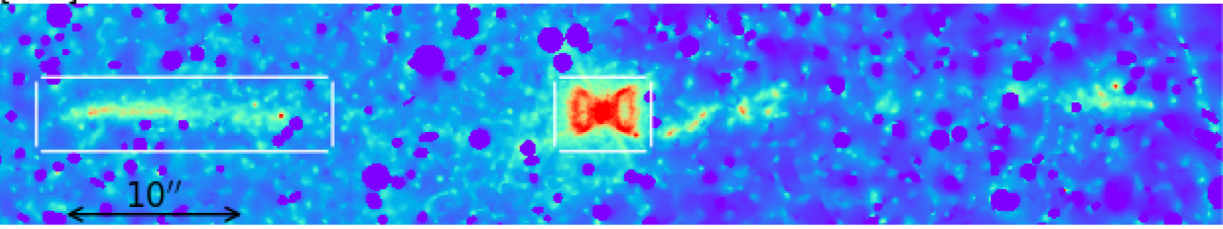

$\mathrm{H} \alpha$
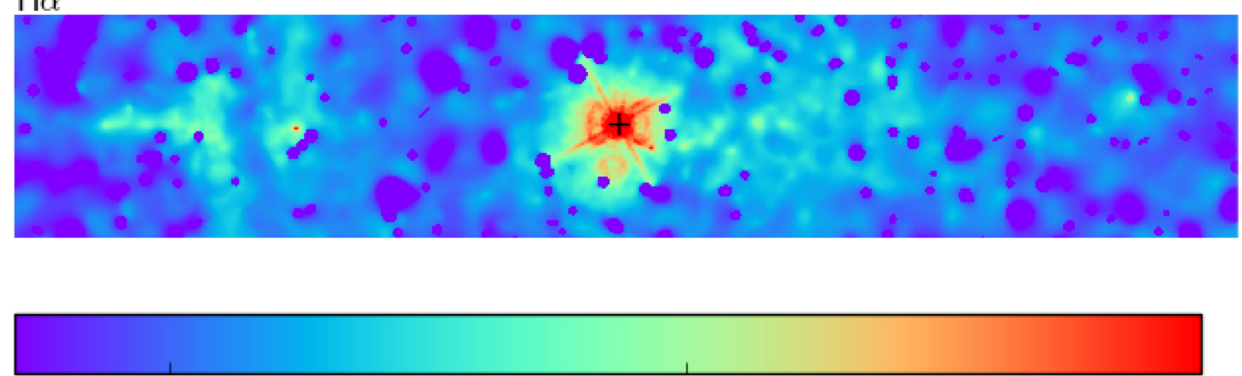

$10^{-16}$

$10^{-15}$

\subsection{The source region}

Figure 4 shows the region around the outflow source (this is the region shown with the central, white box on the top frame of Fig. 3). In both the [N II] and the $\mathrm{H} \alpha$ frames, the source region shows a bipolar cavity structure, aligned with the direction of the outflow. The two cavities appear to end at an elliptical "rim", and together they result in an "hourglass" morphology.

We have carried out paraboloidal fits to all of the peaks in the [N II] frame with fluxes $\geq 3 \times 10^{-15} \mathrm{erg} \mathrm{cm}^{-2} \mathrm{~s}^{-1} \operatorname{arcsec}^{-2}$, and many of the resulting positions (shown as crosses in the top frame of Fig. 4) appear to trace the two elliptical rims. We have then taken all of the intensity peaks with distances from the source along the outflow axis $-1.6^{\prime \prime} \leq x \leq-0.5^{\prime \prime}$ (to the $\mathrm{E}$ of the source) and $0.5^{\prime \prime} \leq x \leq 1.6^{\prime \prime}$ (to the $\mathrm{W}$ ) and have fitted their positions with two ellipses.

In Fig. 5, we show the points which have been used and the resulting elliptical fits. In order to carry out these fits, we have $(0.437 \pm 0.026)^{\prime \prime}$,
Fig. 2. Intermediate steps for obtaining the $[\mathrm{N} \mathrm{II}]$ smoothed image. From top to bottom panels: original [N II] image with masked stars (top panel), bins obtained with Voronoi tessellation (coloured to distinguish them), resulting bin size map and finally smoothed image (bottom panel).

Fig. 3. Smoothed [N II] (top panel) and $\mathrm{H} \alpha$ (bottom panel) images. The scale of the image and two boxes (of the regions covered in Figs. 4 and 6) are shown on the top frame. The cross in the centre of the bottom frame indicates the position of Sanduleak's star. The images are shown with the logarithmic colour scale given (in erg s $\mathrm{cm}^{-1} \mathrm{arcsec}^{-2}$ ) by the bottom bar.

considered ellipses with axes parallel to the coordinate axes of the frames, and have allowed arbitrary centres and major/minor axes. The results of these fits are as follows:

- E ellipse: central position $\left(x_{0}, y_{0}\right)=(-1.039 \pm$ $0.022,0.024 \pm 0.035)^{\prime \prime}$, major axis $a=(1.049 \pm 0.033)^{\prime \prime}$ and minor axis $b=(0.366 \pm 0.005)^{\prime \prime}$,

- W ellipse: central position $\left(x_{0}, y_{0}\right)=(1.058 \pm 0.011,0.047 \pm$ $0.070)^{\prime \prime}$, major axis $a=(1.158 \pm 0.095)^{\prime \prime}$ and minor axis $b=$

giving remarkably similar values for the two outflow lobes.

If we now assume that the two cavity rims are circular, and that their ellipticity is only due to a straightforward projection effect, we can compute the angle between the outflow axis and the plane of the sky as $\phi=\sin ^{-1}(b / a)=(20.6 \pm 0.9)^{\circ}$ (corresponding to the weighted average of the angles obtained from the two elliptical fits). This orientation angle is close to the $\sim 15^{\circ}$ assumed by Angeloni et al. (2011). 
[N II]

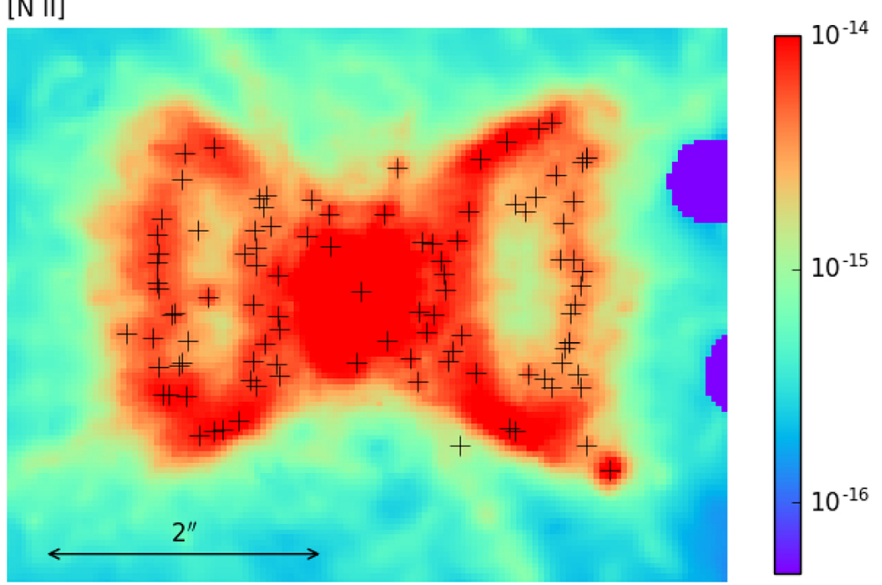

$\mathrm{H} \alpha$

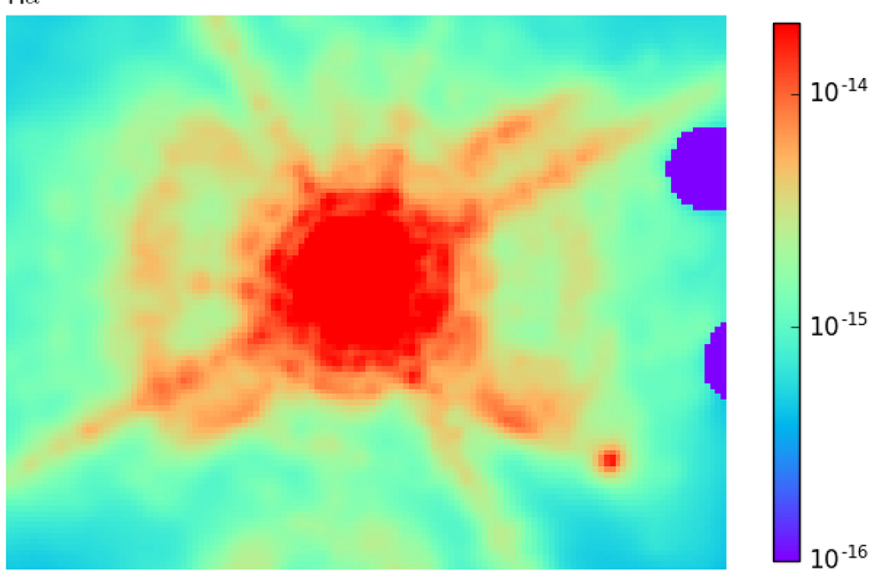

Fig. 4. [N II] (top panel) and $\mathrm{H} \alpha$ (bottom panel) maps of the region around Sanduleak's star (this is the region shown by the central, white box in Fig. 3). The images are shown with the logarithmic colour scales given (in erg s$~^{-1} \mathrm{~cm}^{-2} \operatorname{arcsec}^{-2}$ ) by bars on the right. Diffraction spikes are visible around Sanduleak's star in the two images.

\subsection{The jets}

The $[\mathrm{N} \mathrm{II}]$ emission of the jet/counterjet structure (top frame of Fig. 3) shows a subtle, large-scale curvature, which Angeloni et al. (2011) attribute to a possible precession of the outflow axis. However, it is unclear whether the observed curvature has the "point symmetry" required for a precession, or whether it has a "mirror symmetry" as expected for an orbital motion of the outflow source (see, e.g., Raga et al. 2009).

As the $\mathrm{E}$ jet is better defined, we now concentrate on this outflow lobe. Figure 6 shows the [N II] emission within the Eastern white box in the top frame of Fig. 3. In this zoom of the E jet, we see that there are two apparent "curvatures" (or "deviations") from a straight outflow: one over the whole emitting region (within Fig. 6) with a scale of $\sim 15^{\prime \prime}$, and also smaller scale deviations with a characteristic size of $\sim 2^{\prime \prime}$.

In order to estimate the density of the E jet, we proceed as follows. In the unsmoothed $\mathrm{H} \alpha$ frame (top frame of Fig. 1), the brightest region of the $\mathrm{E}$ jet has an intensity $I_{H \alpha} \approx 3.5 \times$ $10^{-15} \mathrm{erg} \mathrm{s}^{-1} \mathrm{~cm}^{-2} \operatorname{arcsec}^{-2}$ with a full width (perpendicular to the outflow axis) $d \approx 0.2^{\prime \prime}\left(=1.5 \times 10^{17} \mathrm{~cm}\right.$ at $\left.50 \mathrm{kpc}\right)$. This gives an $r_{j}=d / 2=7.5 \times 10^{16} \mathrm{~cm}$ estimate for the jet radius. Using the case $\mathrm{B}$ hydrogen recombination cascade emission coefficient
$j_{\mathrm{H} \alpha} / n_{\mathrm{H}}^{2}=2.83 \times 10^{-26} \mathrm{erg} \mathrm{s}^{-1} \mathrm{~cm}^{3} \operatorname{sterad}^{-1}$ (at $10^{4} \mathrm{~K}$ ), we then obtain an $\mathrm{H} \alpha$ emission measure:

$\mathrm{EM}=\int n_{\mathrm{H}}^{2} \mathrm{~d} l=1700 \mathrm{pc} \mathrm{cm}^{-6}$.

Assuming that the width of the jet along the line of sight has the same value $d$, and a homogeneous $n_{\mathrm{H}}$ within the jet, we obtain

$n_{\mathrm{H}} \approx \sqrt{\frac{\mathrm{EM}}{d}}=190 \mathrm{~cm}^{-3}$.

Finally, we take the E jet region of Fig. 6, and compute the total $\mathrm{H} \alpha$ and $[\mathrm{N} \mathrm{II]} \mathrm{fluxes} \mathrm{(subtracting} \mathrm{a} \mathrm{uniform} \mathrm{background}$ calculated with 10 pixel wide strips along the $\mathrm{N}$ and $\mathrm{S}$ edges). From these fluxes, we obtain line luminosities $L_{H \alpha}=0.07 L_{\odot}$ and $L_{[\mathrm{N} \mathrm{II}]}=0.19 L_{\odot}($ assuming a distance of $50 \mathrm{kpc}$ and no extinction to the $\mathrm{LMC}$ ).

\subsection{The derived parameters}

The determination of a $\phi=21^{\circ}$ orientation angle between Sanduleak's star outflow and the plane of the sky (see Sect. 2.3) allows us to calculate

- from the $29^{\prime \prime}$ projected angular size of the E jet (see Sect. 2.2), a total deprojected length $L=2.3 \times 10^{19} \mathrm{~cm}$,

- from the $360 \mathrm{~km} \mathrm{~s}^{-1}$ mean radial velocity for the heads of the two lobes obtained by Angeloni et al. (2011) from a long-slit spectrum, a deprojected jet velocity $v_{j}=1004 \mathrm{~km} \mathrm{~s}^{-1}$.

These length and velocity estimates give a dynamical timescale $t_{\text {dyn }}=L / v_{j} \approx 7300 \mathrm{yr}$.

The $n_{\mathrm{H}}=190 \mathrm{~cm}^{-3}$ density and $r_{j}=7.5 \times 10^{16} \mathrm{~cm}$ estimates (see Sect. 2.3) then allow us to obtain the mass loss rate:

$\dot{M}_{j}=\left(1.4 m_{\mathrm{H}} n_{\mathrm{H}}\right) \pi r_{j}^{2} v_{j}=1.2 \times 10^{-5} M_{\odot} \mathrm{yr}^{-1}$,

and the mechanical luminosity

$L_{\mathrm{m}}=\frac{1}{2} \dot{M}_{j} v_{j}^{2}=1000 L_{\odot}$.

Our present estimate of the mass loss rate (Eq. (3)) is in surprisingly good agreement with the $\dot{M} \sim 10^{-5} M_{\odot} \mathrm{yr}^{-1}$ of Shore et al. (1987), also discussed by Michalitsianos et al. (1989), which was obtained for the envelope around Sanduleak's star. Our estimate of the mechanical luminosity (Eq. (4)) implies that only a fraction of $\sim 2 \times 10^{-4}$ of the available kinetic energy is radiated as $\mathrm{H} \alpha$ and [N II] line emission (see Sect. 2.4).

\section{Numerical simulations}

\subsection{Conical wind models}

As an exploration of possible dynamical models for Sanduleak's jet, we have computed axisymmetric simulations of a conical outflow with an opening angle that grows as a function of time, travelling into a uniform environment. This flow configuration has been modelled analytically by Cantó et al. (2008).

The simulations are carried out in a four-level, binary adaptive grid with a maximum resolution (along the axial and radial directions) of $2.15 \times 10^{16} \mathrm{~cm}$, using an axisymmetric version of the yguazú-a gasdynamical code (Raga et al. 2000). This version of the code integrates the gasdynamic equations together with one advection/rate equation for ionized hydrogen, and includes a parametrized cooling rate computed with the temperature, density, and ionization fraction of the gas (described in detail by Masciadri \& Raga 2001a). 


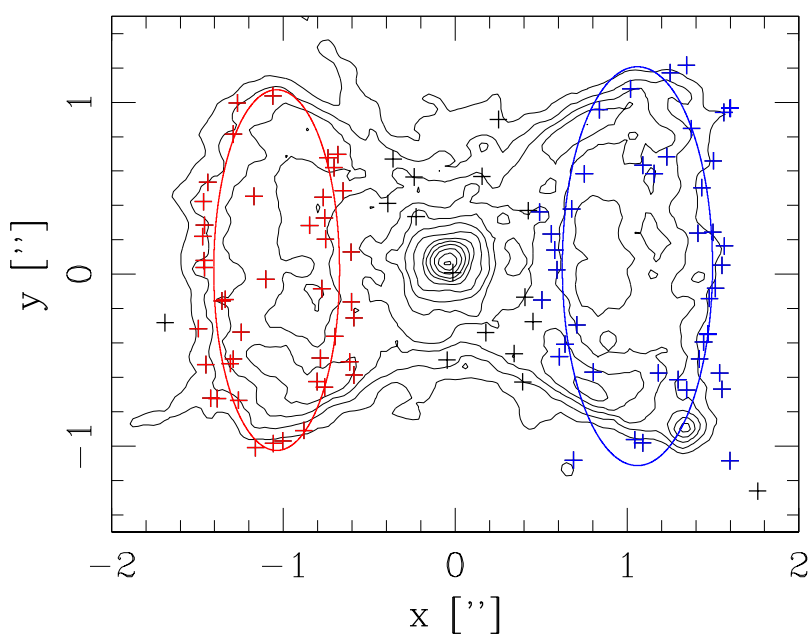

Fig. 5. Contour plot of the $[\mathrm{N} \mathrm{II}]$ emisison of the region around Sanduleak's star (shown with logarithmic, factor of 2 contours). The crosses indicate the local maxima found in the image, and the red and blue crosses are the values chosen for the elliptical fits (see the text). The resulting ellipses are shown by the red and blue curves (corresponding to the red and blue outflow lobes). The axes are labeled with offsets (in arcsec) from Sanduleak's star, along and across the outflow axis.

The simulations are initialized with a uniform, neutral environment of number density $n_{a}=1 \mathrm{~cm}^{-3}$ and temperature $T_{a}=$ $1000 \mathrm{~K}$ filling the computational grid. The jet inflow is imposed in a cone of initial half-opening angle $\alpha_{0}=2^{\circ}$ (this angle is then allowed to increase as a function of time, see below) and axial length $R_{0}=10^{18} \mathrm{~cm}$, filled with a radial flow of velocity $v_{j}=1000 \mathrm{~km} \mathrm{~s}^{-1}$. The jet material at the outer limit of the cone has a density $n_{j}=1000 \mathrm{~cm}^{-3}$ and a temperature $T_{j}=1000 \mathrm{~K}$. The jet density is then lowered as a function of time so as to keep a constant $\dot{M}_{j}=1.3 \times 10^{-5} M_{\odot} \mathrm{yr}^{-1}$ mass loss rate within the opening-out cone.

We compute two models:

- W1: a conical jet with a half-opening angle that at $t=0$ has a value $\alpha_{0}=2^{\circ}$, and for $t>0$ grows at a rate of $6^{\circ} / 1000 \mathrm{yr}$,

- W2: a conical jet with a constant half-opening angle $\alpha_{0}=2^{\circ}$

for $t<4000 \mathrm{yr}$, and which for larger $t$ opens out at a constant rate of $8.2^{\circ} / 1000 \mathrm{yr}$,

letting them evolve to a time $t=6000 \mathrm{yr}$, in which the head of the lobe has reached a distance of $\sim 2 \times 10^{19} \mathrm{~cm}$ from the outflow source.

Figure 7 shows the $t=6000 \mathrm{yr}$ density stratifications obtained from models W1 and W2. Model W1 shows a conical flow close to the outflow source, with wings that first open out and then converge (at larger distances from the source) to an axial "plug" of dense material that drives the head of the lobe. Model W2 illustrates the fact that the axial plug of dense material can be lengthened by initially keeping a constant opening angle (of the outflow cone) for a given time interval, before allowing it to increase with time.

\subsection{Jet+stratified envelope models}

We have also computed two models of an initially cylindrical jet, travelling into a stratified environment. The environment has a spherical density profile of $n \propto R^{-2}$ (where $R$ is the spherical radius measured from the outflow source). The density stratification is normalized to a value of $1 \mathrm{~cm}^{-3}$ at a radius $R_{0}=5 \times 10^{18} \mathrm{~cm}$. For spherical radii $R>R_{0}$, the environmental density is uniform, with a value of $1 \mathrm{~cm}^{-3}$. The neutral environment has a uniform temperature of $1000 \mathrm{~K}$.
The jet flow is imposed in a cylinder of $10^{17} \mathrm{~cm}$ radius and $2 \times 10^{17} \mathrm{~cm}$ length. The (initially neutral) jet has a density $n_{j}=$ $100 \mathrm{~cm}^{-3}$ and a temperature $T_{j}=1000 \mathrm{~K}$.

With this setup, we compute two models:

- J1: with a constant injection velocity $v_{j}=1000 \mathrm{~km} \mathrm{~s}^{-1}$,

- J2: with an initial velocity $v_{j, 0}=1000 \mathrm{~km} \mathrm{~s}^{-1}$, which then

decreases linearly with time reaching $v_{j}=0$ at $\tau_{j}=3000 \mathrm{yr}$

(and remaining at $v_{j}=0$ for $t>\tau_{j}$ ).

With these parameters, model $\mathrm{J} 1$ has a time-independent mass loss rate $\dot{M}_{j}=1.1 \times 10^{-5} M_{\odot} \mathrm{yr}^{-1}$ and model J 2 initially has this value of $\dot{M}_{j}$, which then decreases linearly with time.

Figure 8 shows the $t=7000 \mathrm{yr}$ model $\mathrm{J} 1$ and $t=8000 \mathrm{yr}$ model $\mathbf{J} 2$ density stratifications. Both models have an opening out, walled cavity close to the outflow source. Model J1 has a jet of approximately uniform density (with a value similar to the injection density), ending in a high-density "head" of piled up jet and environmental material. Model J2 has a similar "head", but has a density that decreases from the head toward the outflow source.

\section{Discussion}

We have used archival HST images of the jet system of Sanduleak's star (in the LMC) to determine:

- a $\phi \approx 21^{\circ}$ angle between the outflow axis and the plane of the sky (see Sect. 2.3),

- a $v_{j} \approx 1000 \mathrm{~km} \mathrm{~s}^{-1}$ peak jet velocity (deprojecting the radial velocities of Angeloni et al. 2011, see Sect. 2.4),

- a length $L \approx 2.3 \times 10^{19} \mathrm{~cm}$ (see Sect. 2.5$)$,

- a radius $r_{j} \approx 7.5 \times 10^{16} \mathrm{~cm}$ (see Sect. 2.4),

- a $\approx 200 \mathrm{~cm}^{-3}$ number density,

- a mass loss rate $\dot{M}_{j} \approx 1.2 \times 10^{-5} M_{\odot} \mathrm{yr}^{-1}$,

- a mechanical luminosity $L_{\mathrm{m}} \approx 1000 L_{\odot}$ (see Sect. 2.5),

- an $\mathrm{H} \alpha+[\mathrm{N} \mathrm{II}]$ luminosity $\approx 0.26 L_{\odot}$,

using mostly the $\mathrm{E}$ jet of the bipolar outflow.

With these parameters we have then computed numerical simulations of:

- a "conical wind" with a half-opening angle $\alpha$ that increases with time, moving into a uniform environment,

- a "cylindrical jet" (i.e., with 0 opening angle) moving into a stratified core with a density decreasing as $1 / R^{2}$, and eventually merging into a uniform environment.

For the conical wind scenario (see Fig. 7), we have computed simulations with an $\alpha$ that increases linearly with time, and with an $\alpha=2^{\circ}$ that is constant for $4000 \mathrm{yr}$ and then increases linearly for larger times. The simulations show that this scenario is able to produce an "opening out" structure with a curved outer boundary that at larger distances recollimates and joins an axial "plug" of dense material (the length of which can be adjusted by choosing an appropriate $\alpha$ vs. time law, as illustrated by the W1 and W2 models, see Fig. 7). This structure is reminiscent of the W jet from Sanduleak's star (see Fig. 3) which appears to have emission partially joining the $S$ wall of the source cavity and the jet. This "opening out wind" scenario, however, does not produce a structure around the source with denser "walls", as apparently implied by the [N II] and $\mathrm{H} \alpha$ emission of the region around Sanduleak's star (see Figs. 4 and 5).

For the jet+stratified core scenario (see Fig. 8), we have computed simulations of a constant velocity jet, and of a jet with an ejection velocity that decreases linearly with time. Both simulations produce an opening-out, low-density cavity surrounded by a high-density shell around the source position. This dense shell at larger distances joins the leading bow shock of the jet head. The fact that an "hourglass" shell structure is produced in 

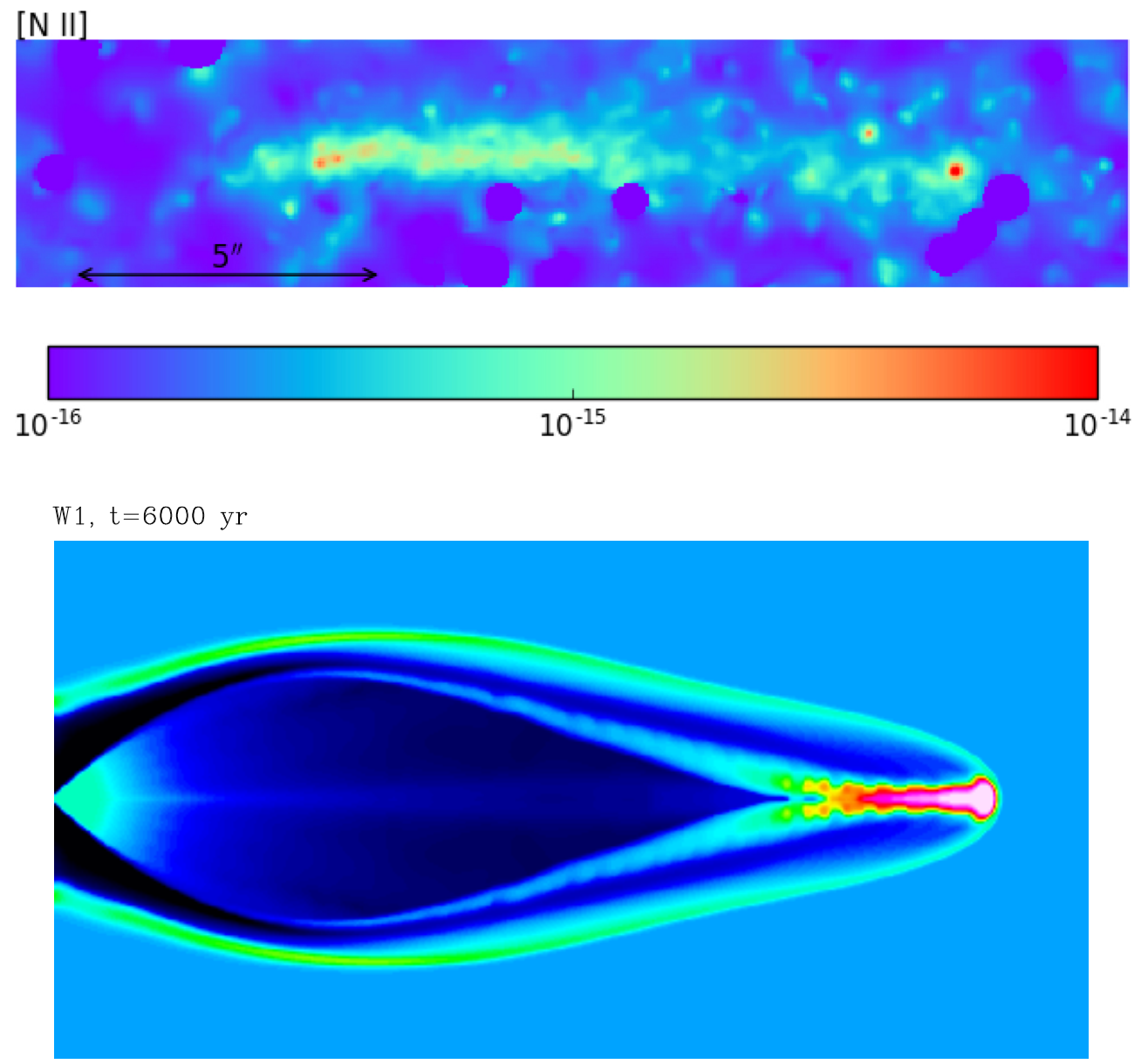

W2, $t=6000 \mathrm{yr}$

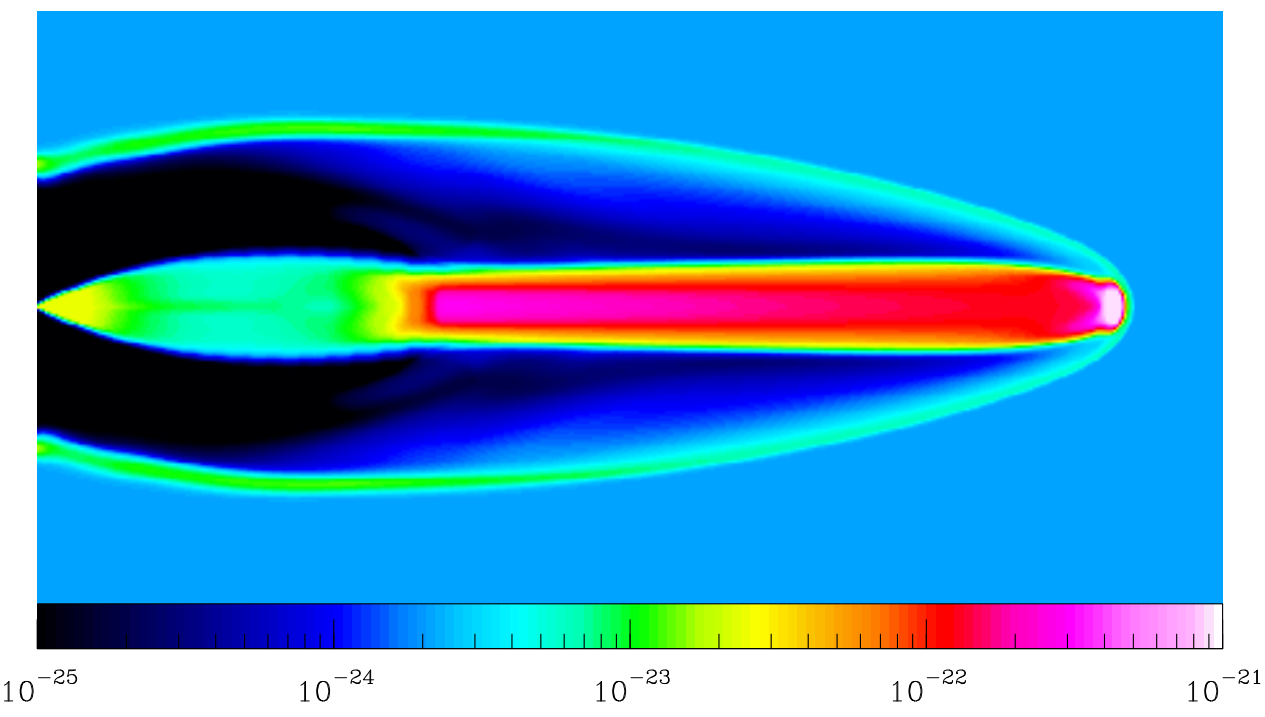

Fig. 6. [N II] map of the E jet shown with the logarithmic colour scale given (in $\mathrm{erg} \mathrm{s}^{-1} \mathrm{~cm}^{-2} \operatorname{arcsec}^{-2}$ ) by the bottom bar. This graph shows the region within the $\mathrm{E}$ box shown (in white) in the top frame of Fig. 3.
Fig. 7. Density stratifications at $t=$ $6000 \mathrm{yr}$ obtained from the conical wind models W1 (top panel) and W2 (bottom panel). The frames have an axial extent of $2.1 \times 10^{19} \mathrm{~cm}$. The radial axis of the axisymmetric simulations has been reflected. The densities are shown with the logarithmic colour scale shown by the bottom bar (in $\mathrm{g} \mathrm{cm}^{-3}$ ). this scenario is in qualitatively better agreement with the limbbrightened cavity seen around Sanduleak's star (see Figs. 4 and 5). However, the observations do not show an extension of the observed shell to the leading region of the jets (see Fig. 3). Also, the constant velocity jet simulation ( $\mathrm{J} 1$, top frame of Fig. 8) has a jet beam extending from the head to the position of the source, which is clearly not seen in the observed jets. This problem is overcome imposing a time-dependent ejection velocity (e.g., the one of model J2), so that the jet is cut off at later evolutionary times (see the bottom frame of Fig. 8).
Therefore, both of the scenarios that we have explored have characteristics that resemble the observations of the outflow from Sanduleak's star. It will possibly be necessary to include features of both of these scenarios in order to obtain a convincing model for the jet from Sanduleak's star.

The comparison between the models and the observations is of course only tentative, since we have not computed emission line maps from the simulations. In order to do this, it will be necessary to find which is the mechanism that heats/ionizes the emitting gas. This could be done through: 


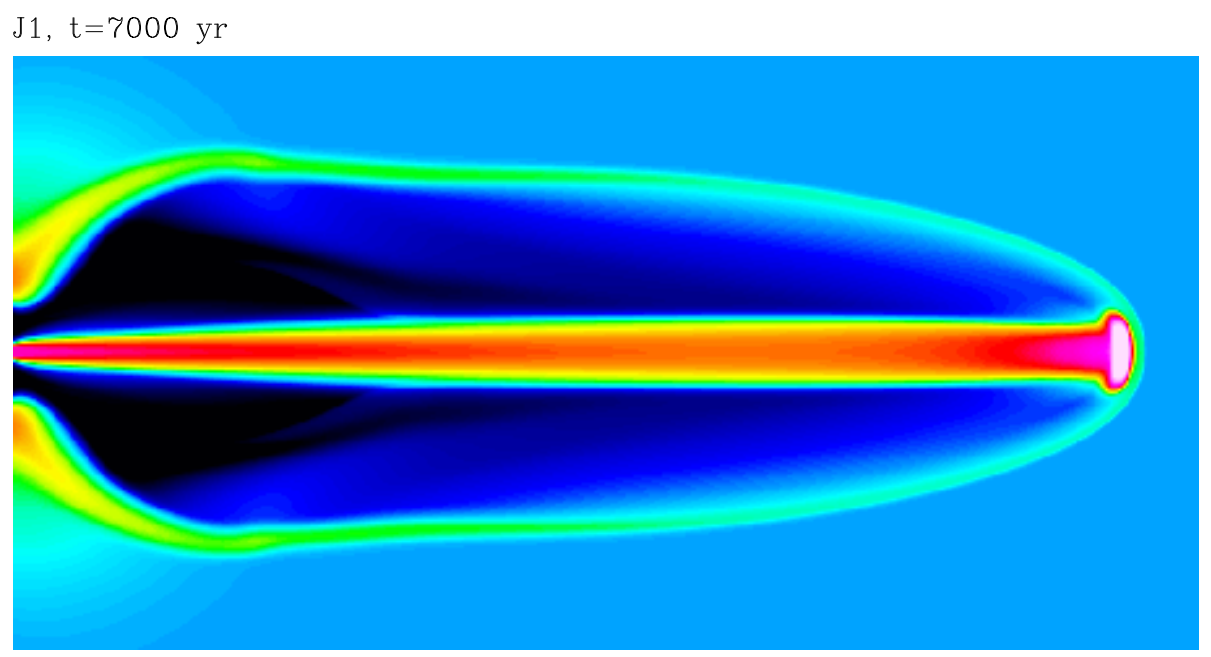

$$
\mathrm{J} 2, \mathrm{t}=8000 \mathrm{yr}
$$

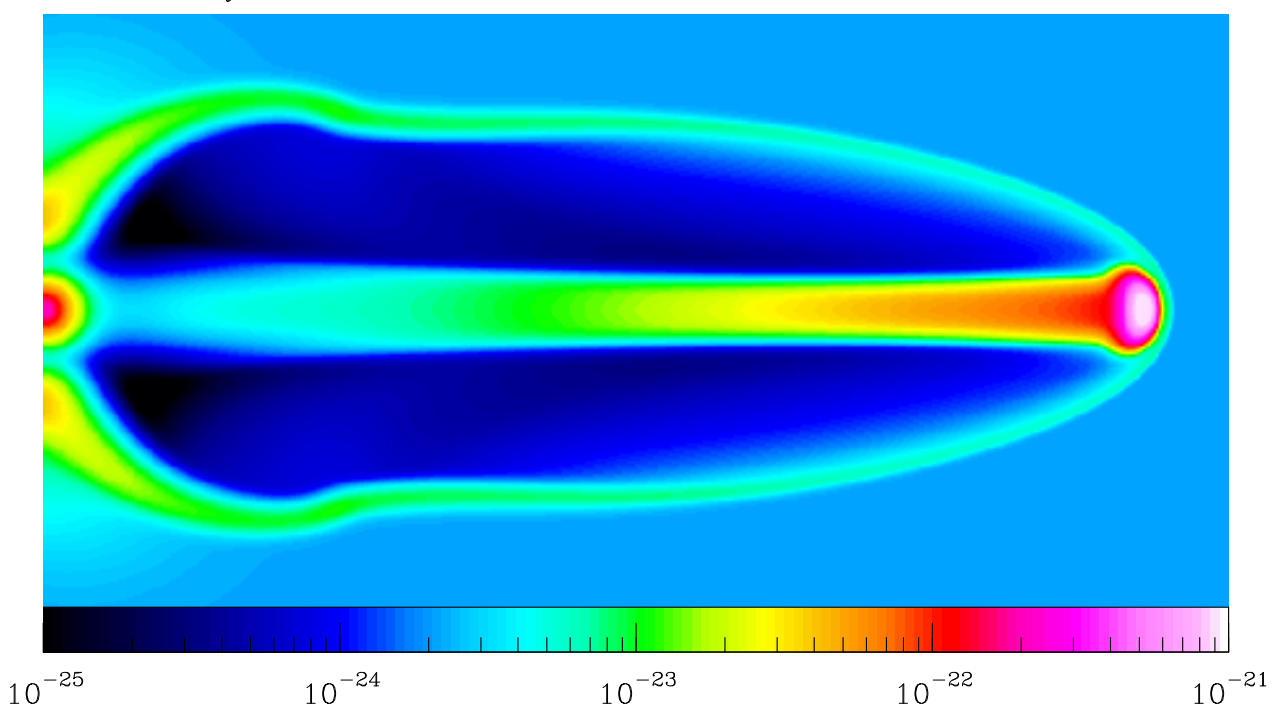

Fig. 8. Density stratifications obtained from the cylindrical jet models J1 (top panel, at $t=7000 \mathrm{yr}$ ) and $\mathrm{J} 2$ (bottom panel, at $t=8000 \mathrm{yr}$ ). The frames have an axial extent of $2.1 \times 10^{19} \mathrm{~cm}$. The densities are shown with the logarithmic colour scale shown by the bottom bar (in $\mathrm{g} \mathrm{cm}^{-3}$ ).
- photoionization due to the presence of a hot star in the possibly binary source of the system,

- tapping the kinetic luminosity of the outflow (e.g., through turbulent mixing and/or shocks produced by short period variations in the outflow velocity).

In their present version, our numerical simulations produce emission line maps which are dominated by the leading head of the outflow lobes, which is definitely not in agreement with the observations.

Another characteristic that should be addressed by theoretical models are the deviations from axisymmetry of the observed outflow. This jet does not show the clear, pointsymmetric structures of some jets from evolved stars, such as Fleming 1 (see, e.g., Palmer et al. 1996) and R Aquarii (see, e.g., Melnikov et al. 2018). In the case of the jet from Sanduleak's star, it is not even completely clear whether the observed sinuous structures are point-symmetric (as expected from a precession) or mirror symmetric, as expected in jets from sources with a fast orbital motion (see, e.g., Masciadri \& Raga 2002).

In order to model the deviations from axisymmetry (actually, from "side-to-side", projected symmetry) observed in the jets from Sanduleak's star, there are at least two possible scenarios that should be explored:
- the presence of a precession of the ejection and/or an orbital motion of the source. These possibilities would imply the presence of a binary (or multiple) system, producing the orbital motion of the source and/or the precession of a disk structure around the source (see, e.g., Raga et al. 2009),

- a motion of Sanduleak's star with respect to a (relatively dense) surrounding environment.

While the "binary source" scenario can produce structures with point and/or mirror symmetries, a motion of the source (relative to the surrounding environment) will produce a side-to-side asymmetry (across the outflow axis, resulting from the motion of the source across this axis) as well as differences between the two outflow lobes (produced by the motion of the source parallel to the outflow axis).

Future progress in modelling this jet system will hinge on further observations. Both high-resolution Fabry-Pérot and "integral field unit" spectroscopic observations of this outflow should give a much clearer idea of the nature of this outflow. Also, high angular resolution infrared observations should clarify the nature of the circumstellar structure around Sanduleak's star. These observations will not be straightforward because of the faintness of the observed emission. However, they might provide substantial progress in the understanding of the bipolar jet from Sanduleak's star and of collimated jets 
from massive stars in general, see, e.g., Friedjung (2011) and Shore (2013).

Acknowledgements. We acknowledge support from the DGAPA-UNAM grants IG100218 and IN109518. We thank an anonymous referee for helpful comments.

\section{References}

Allen, D. A. 1980, ApJ, 20, L131

Angeloni, R., Di Mille, F., Bland-Hawthorn, J., \& Osip, D. J. 2011, ApJ, 743, L8

Bertin, E., \& Arnouts, S. 1996, A\&AS, 117, 393

Bugarella, D., Vogel, M., \& Paresce, F. 1992, A\&A, 262, 83

Cantó, J., Raga, A. C., \& Williams, D. A. 2008, Rev. Mex. Astron. Astrofis., 44, 293

Cappellari, M., \& Copin, Y. 2003, MNRAS, 342, 345

Friedjung, M. 2011, A\&A, 536, A97

Harvey, E., Redman, M. P., Boumis, P., \& Akras, S. 2016, A\&A, 595, A64

Kafatos, M., Michalitsianos, A. G., Allen, D. A., \& Stencel, R. E. 1983, ApJ, 275,584
Lloyd, H. M., Bode, M. F., O’Brien, T. J., \& Kahn, F. D. 1993, MNRAS, 265, 457

Masciadri, E., \& Raga, A. C. 2001a, A\&A, 376, 1073

Masciadri, E., \& Raga, A. C. 2002b, ApJ, 568, 733

McLeod, A. F., Reiter, M., Kuiper, R., Klaasen, P. D., \& Evans, C. J. 2018, Nature, 554, 334

Meaburn, J., Gehring, G., Walsh, J. R., et al. 1993, A\&A, 276, L21

Melnikov, S., Stute, M., \& Eislöffel, J. 2018, A\&A, 612, A77

Michalitsianos, A. G., Kafatos, M., \& Shore, S. N. 1989, ApJ, 341, 367

Palmer, J. W., López, J. A., Meaburn, J., \& Lloyd, H. M. 1996, A\&A, 307, 225

Raga, A. C., Navarro-González, R., \& Villagrán-Muniz, M. 2000, Rev. Mex. Astron. Astrofis., 36, 67

Raga, A. C., Esquivel, A., Velázquez, P. F., et al. 2009, ApJ, 707, L6

Sanduleak, N. 1977, IBVS, 1304, 1

Schaefer, B. E. 2008, AJ, 135, 112

Shore, S. 2013, A\&A, 559, L7

Shore, S. N., Sanduleak, N., \& Allen, D. A. 1987, A\&A, 176, 59

Soker, N. 2005, ApJ, 619, 1064

Velusamy, T., \& Langer, W. D. 1998, Nature, 392, 685 